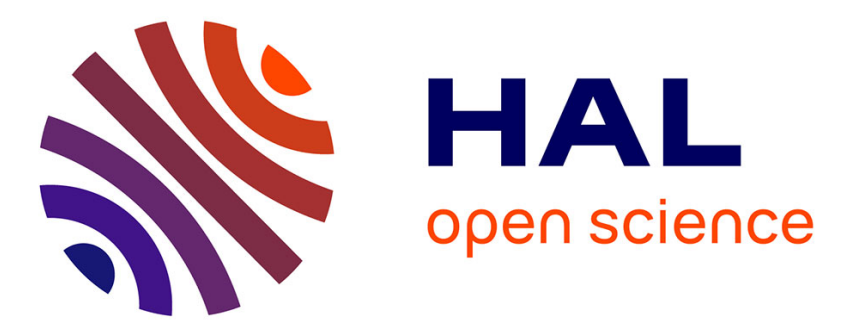

\title{
Assessment of Axial Wave Number and Mean Flow Uncertainty on Acoustic Liner Impedance Eduction
}

\author{
Douglas M. Nark, Michael G. Jones, Estelle Piot
}

\section{To cite this version:}

Douglas M. Nark, Michael G. Jones, Estelle Piot. Assessment of Axial Wave Number and Mean Flow Uncertainty on Acoustic Liner Impedance Eduction. AIAA/CEAS Aeroacoustics Conference, 2018, Jun 2018, ATLANTA, United States. pp.1-12, 10.2514/6.2018-3444 . hal-02340220

\author{
HAL Id: hal-02340220 \\ https://hal.science/hal-02340220
}

Submitted on 30 Oct 2019

HAL is a multi-disciplinary open access archive for the deposit and dissemination of scientific research documents, whether they are published or not. The documents may come from teaching and research institutions in France or abroad, or from public or private research centers.
L'archive ouverte pluridisciplinaire HAL, est destinée au dépôt et à la diffusion de documents scientifiques de niveau recherche, publiés ou non, émanant des établissements d'enseignement et de recherche français ou étrangers, des laboratoires publics ou privés. 


\title{
Assessment of Axial Wave Number and Mean Flow Uncertainty on Acoustic Liner Impedance Eduction
}

\author{
Douglas M. Nark* and Michael G. Jones ${ }^{\dagger}$ \\ NASA Langley Research Center, Hampton, VA 23681-2199, U.S.A \\ Estelle Piot ${ }^{\ddagger}$ \\ ONERA/DMPE, Université de Toulouse, F-31055, Toulouse, France
}

\begin{abstract}
A key parameter in designing and assessing advanced broadband acoustic liners to achieve the current and future noise reduction goals is the acoustic impedance presented by the liner. This parameter, intrinsic to a specific liner configuration, is dependent on sound pressure level and grazing flow velocity. Current impedance eduction approaches have, in general, provided excellent results and continue to be employed throughout the acoustic liner community. However, some recent applications have indicated a possible dependence of the educed impedance on the direction of incident waves relative to the mean flow. The purpose of the current study is to investigate this unexpected behavior for various impedance eduction methods based on the Pridmore-Brown and convected Helmholtz equations. Specifically, the effects of flow profile and axial wavenumber uncertainties on educed impedances for upstream and downstream sources are investigated. The uniform flow results demonstrate the importance of setting a correct Mach number value in obtaining consistent educed impedances for upstream and downstream sources. In fact, the consistency of results over the two source locations was greatly improved by a slight modification of the uniform flow Mach number. In addition, uncertainty in educed axial wavenumber was also illustrated to correlate well with differences in the educed impedances, even with modified uniform flow Mach number. Finally, while less straightforward than in the uniform flow case, it appears that modification of the mean flow profile may also improve consistency of results for upstream and downstream results when shear flow is included.
\end{abstract}

\section{Nomenclature}

$c_{o}$

$d$

$d_{h}$

$H$

$k_{m n}$

$M_{o}$

$p$

$t$

Symbols:

$\zeta$

$\theta$

$\lambda$

$\rho_{o}$

$\chi$

sound speed

liner core depth

liner perforate facesheet hole diameter

duct height; for GFIT $H=2.5$ in. $(63.5 \mathrm{~mm})$

axial wave number

mean flow Mach number

acoustic pressure

liner perforate facesheet thickness

* Senior Research Scientist, Structural Acoustics Branch, Research Directorate, AIAA Associate Fellow

${ }^{\dagger}$ Senior Research Scientist, Structural Acoustics Branch, Research Directorate, AIAA Associate Fellow

${ }^{\ddagger}$ Research Scientist 
Subscripts:

$m, n$

horizontal and vertical mode numbers

$\begin{array}{ll}\text { Abbreviations: } & \\ \text { CHE } & \text { Convected Helmholtz Equation } \\ \text { GFIT } & \text { Grazing Flow Impedance Tube } \\ \text { PBE } & \text { Pridmore-Brown Equation } \\ \text { SPL } & \text { Sound Pressure Level }\end{array}$

\section{Introduction}

Aircraft noise reduction requirements continue to become more stringent with increases in air traffic. As fan noise produced by the engine is a dominant noise source for subsonic aircraft, significant effort has been directed toward the reduction of this component both at its source and along transmission/radiation paths. Acoustic liners have been particularly effective at reducing fan noise transmission/radiation. However, the introduction of advanced fan designs and shorter engine nacelles has highlighted a need for novel acoustic liner designs that provide increased fan noise reduction over a broader frequency range.

A key parameter in designing and assessing advanced broadband acoustic liners to achieve the aforementioned noise reduction goals is the acoustic impedance presented by the liner. This parameter, intrinsic to a specific liner configuration, is dependent on sound pressure level and grazing flow velocity. As discussed by Jones and Watson, 1 a number of methods have been developed to educe acoustic impedance in the presence of grazing flow. These range from direct measurement approaches (e.g., in situ microphones/probes, Laser Doppler Velocimetry) to indirect methods based on the combination of a duct propagation code and measurements within the hardwall sections (upstream/downstream or opposite) of a liner mounted in the flow duct. The indirect approaches have generally been based on three sets of equations: the convected Helmholtz equation, limited to uniform background flow, and the linearized Euler equations and the Pridmore-Brown equation, which include shear-flow effects. 23 The methods based on the convected Helmholtz and linearized Euler equations assume a finite-length acoustic liner mounted in a rectangular waveguide. In this case, the educed impedance value is iterated upon until the acoustic pressures predicted by a duct propagation code match the acoustic pressures measured at a number of microphone locations to within an acceptable tolerance. Alternatively, the method based on the Pridmore-Brown equation 4 assumes an infinite-length acoustic liner mounted in a rectangular waveguide. This approach, which is well suited to cases where a single mode is dominant over a significant portion of the liner, uses amplitude and phase decay rates over the axial extent of the liner to directly compute the liner impedance.

Use of these three impedance eduction approaches implementing the Ingard ${ }^{5}$-Myers 5 boundary condition has, in general, provided excellent results and they continue to be employed throughout the acoustic liner community. However, some recent applications ${ }^{7 \cdot-9}$ have indicated a possible dependence of the educed impedance on the direction of incident waves relative to the mean flow. This unexpected behavior for locally reacting liners has led to the proposal of alternative boundary conditions and to the questioning of the hypotheses underlying the classical impedance eduction methods (see for instance Zhou and Bodén, ${ }^{10}$ Spillere et al., ${ }^{11}$ Bodén et al ${ }^{12}$ ). The purpose of the current study is to investigate the effects of flow profile and axial wavenumber uncertainties on impedance eduction methods based on the Pridmore-Brown and convected Helmholtz equations for upstream and downstream sources. A description of the duct geometry and test samples used in this investigation is provided in Section III. Details on the impedance eduction approaches are offered in Section III. Results are provided in Section IV, and concluding remarks regarding some of the more significant results and further areas of interest are presented in Section $V$.

\section{Description of Test Configurations}

\section{A. Flow Duct}

Over the last three decades, the NASA Langley Research Center has developed a number of test rigs for the evaluation of acoustic liners with and without grazing flow. For this study, the GFIT (see Fig. 1) was selected due to its extensive use in previous impedance eduction work ${ }^{8} 13-15$ and to limit the presence of higher modes in the analysis. The GFIT, where the surface of the test liner forms a portion of the upper wall of the flow duct, has a cross-sectional geometry of 2.0" (50.8 mm) wide by $2.5 "(63.5 \mathrm{~mm})$ high and allows evaluation of acoustic liners with lengths from 2.0 " (50.8 
$\mathrm{mm})$ to 24.0 " (609.6 $\mathrm{mm})$. The test liner is assumed to have an unknown, but uniform, normalized impedance, $\zeta$. Throughout this paper all impedances are normalized by the characteristic impedance, $\rho_{0} c_{0}$, of the air flowing in the duct (left to right in Fig. 11). Acoustic drivers mounted upstream or downstream of the liner sample form the source section for the exhaust or inlet modes, respectively. For this study, these drivers are used to generate tones (one frequency at a time) over a frequency range of 400 to $3000 \mathrm{~Hz}$ in $200 \mathrm{~Hz}$ increments, at source levels (peak total SPL measured near the leading edge of the liner) of 120 and $140 \mathrm{~dB}$, and at centerline Mach numbers of $0.0,0.3$, and 0.5 (measured via pitot-static probe located approximately one inch downstream of the downstream drivers). Fifty-three (53) microphones flush-mounted in the lower wall (opposite the liner, see Fig. 1 p) are used to measure the acoustic pressure field over the axial extent of 40 in $(1.016 \mathrm{~m})$ beginning 8.0 in $(0.203 \mathrm{~m})$ upstream of the liner leading edge.

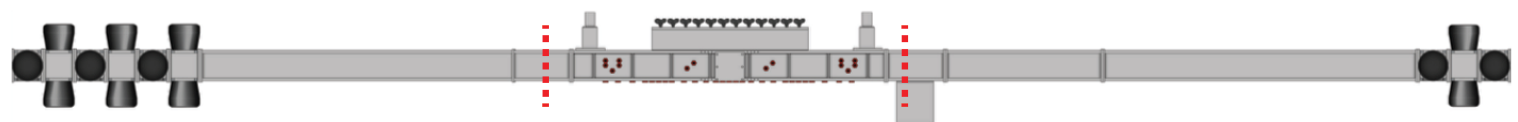

(a) GFIT schematic showing upstream and downstream drivers (exhaust and inlet modes, respectively). Red dashed vertical lines represent upstream and downstream axial locations for mean flow profile measurements.

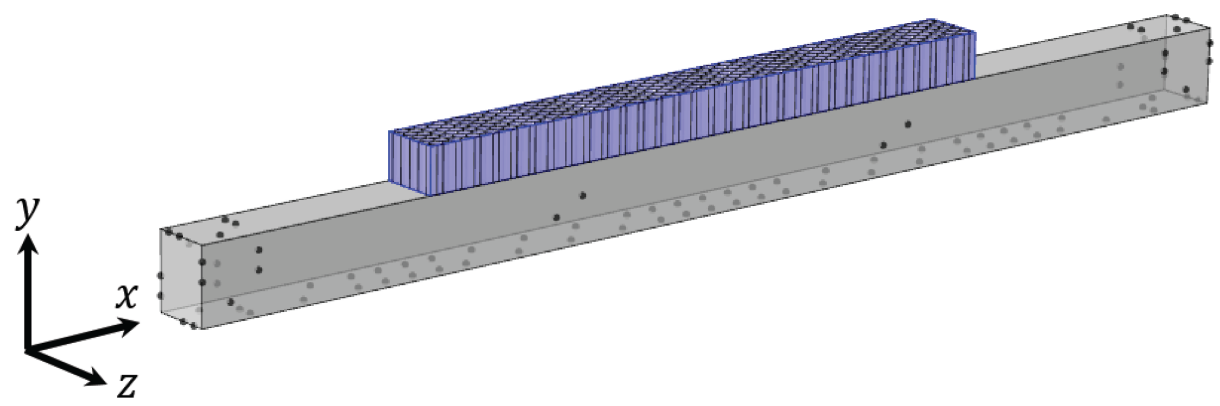

(b) GFIT test section with flush-mounted microphones and liner.

Figure 1: Sketches of the Grazing Flow Impedance Tube (GFIT).

\section{B. Liner Configurations}

Two liner configurations having a length (treated area) of 16 in $(0.406 \mathrm{~m})$ are considered in the current study (see Fig. 22). The first liner contains numerous ceramic tubes with a depth of $3.25 \mathrm{in}(82.55 \mathrm{~mm})$ and a diameter of 0.025 in $(0.635 \mathrm{~mm})$. These tubes combine to provide a surface porosity of $57 \%$. This liner is chosen because it is very linear, i.e., its impedance is independent of source SPL. The impedance of this liner is also insensitive to changes in the tangential flow velocity. The second liner consists of a perforate facesheet bonded to a honeycomb core, which is in turn bonded to a solid backplate. The perforate facesheet has a sheet thickness, $t$, of 0.025 in $(0.635 \mathrm{~mm})$, a hole diameter, $d_{h}$, of $0.039 \mathrm{in}(0.991 \mathrm{~mm})$, and a porosity of $15 \%$. The core depth, $d$, is $1.5 \mathrm{in}(38.1 \mathrm{~mm})$ and the impedance of this conventional perforate-over-honeycomb liner is weakly dependent on changes to the source SPL and tangential flow velocity. Data was acquired in the GFIT for both liners using two sources (upstream and downstream of the liner), two source SPLs $(120,140 \mathrm{~dB}$ ), and three tangential flow velocities (Mach 0.0, 0.3, 0.5). Also, each test condition was repeated eleven times, such that the results can be used for measurement uncertainty analysis.

In addition to acoustic data, 2D flow profiles were acquired upstream and downstream of the test section. These profiles were acquired with each liner installed, as well as a hardwall insert, such that the effects of the liners could be assessed. A total pressure tube was traversed across the duct in 9 horizontal sweeps, where each sweep included 31 locations in the vertical dimension, for a total of 279 total pressures at each axial location. These total pressures were combined with a static pressure measurement in the same axial plane to determine the corresponding mean flow velocities (or Mach numbers). These results were used to compute 1D flow profiles using the 31 points acquired at the spanwise centerline (sweep 5 of 9 ) location, and were also used to compute 2D flow profiles, such that the effects of mean flow profile on the impedance eduction process could be evaluated. In addition, these flow profiles were used 


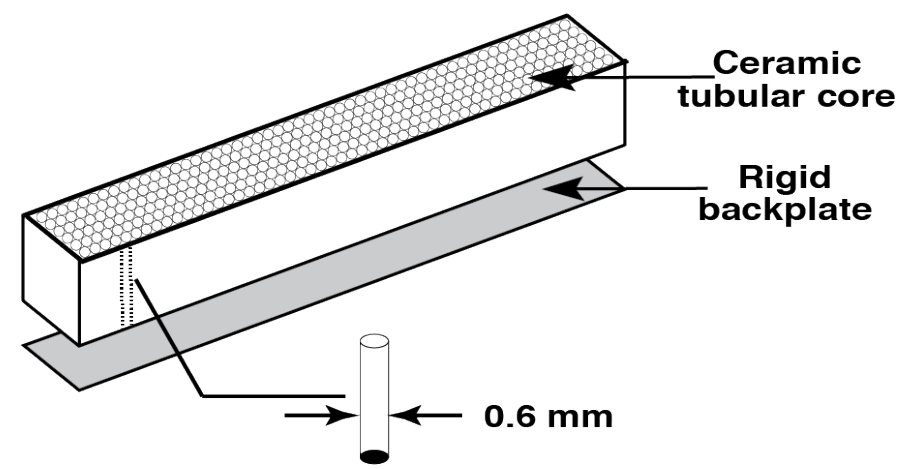

(a) Ceramic tubular (CT57) liner.

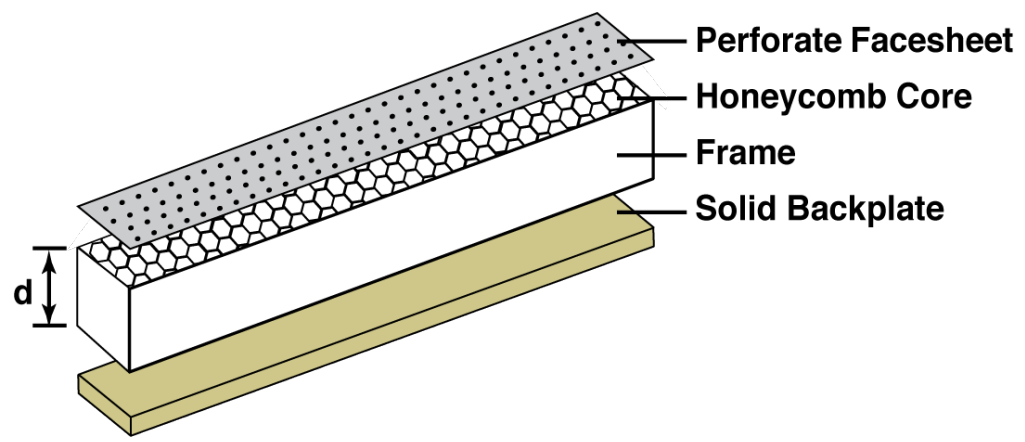

(b) Conventional, perforate-over-honeycomb (GE03) liner.

Figure 2: Sketches of CT57 and GE03 test liners.

to compute average (over all spanwise measurement locations) Mach numbers at both the upstream and downstream axial locations, for use in the uniform flow impedance eduction methods.

\section{Impedance Eduction Approach}

For the current investigation, multiple indirect impedance eduction approaches are used to educe the liner impedance from the measured, multimodal, acoustic pressure field over the test liners. Initially, a method (labeled PBE) based on the Pridmore-Brown equation in the form

$$
P_{m n}^{\prime \prime}(y)+\frac{2 k_{m n} M_{o}^{\prime}(y) P_{m n}^{\prime}(y)}{k-k_{m n} M_{o}(y)}+\left[\left(k-k_{m n} M_{o}(y)^{2}-k_{m n}^{2}-\left(\frac{m \pi}{H}\right)^{2}\right] P_{m n}(y)=0\right.
$$

and implemented by Watson et al $\frac{16}{16}$ is utilized. Here, the superscript / denotes the ordinary derivative with respect to the normal direction, $y$, and $P_{m n}(y)$ and $k_{m n}$ denote the acoustic pressure and associated axial propagation constant for a given horizontal $(m)$ and vertical $(n)$ normal duct mode. The governing equation (Eq. 1 ) is also subject to the boundary conditions

$$
\begin{gathered}
P_{m n}^{\prime}(0)=0 \\
P_{m n}^{\prime}(H)=-\frac{i k}{\zeta}\left[1-k_{m n} M_{o}(H) / k\right]^{2} P_{m n}(H)
\end{gathered}
$$

where Eq. 2 specifies a rigid lower wall and Eq. 3 specifies a treated upper wall using the the Ingard-Myers boundary condition. Under the assumption of an infinite-length acoustic liner mounted in a rectangular waveguide, the axial propagation constants are obtained from measured data. Then, Eqs. 1 and 2, along with specification of $P_{m n}(0)$, may be used to numerically determine $P_{m n}^{\prime}(H)$ and $P_{m n}(H)$. Subsequently, Eq. 3 may be used to educe the unknown impedance, $\zeta$, of the test liner: 


$$
\zeta=-\frac{i k\left[1-k_{m n} M_{o}(H) / k\right]^{2} P_{m n}(H)}{P_{m n}^{\prime}(H)} .
$$

Note that this procedure may be used for either sheared $\left(M_{o}(y)\right)$ or uniform $\left(M_{o}^{\prime}=0\right)$ mean flows. The latter condition will be considered first to isolate the effects of the specified mean flow magnitude.

In addition to the aforementioned PBE approach, an impedance eduction method (labeled CHE) that solves the convective Helmholtz equation using a finite element method ${ }^{13}$ is also used. Here, a finite-length acoustic liner is assumed to be mounted in a rectangular waveguide and the full GFIT test section (see Figure 1) is modeled. The Ingard-Myers impedance boundary condition is incorporated in the CHE analysis in weak form and cubic Hermite polynomials are used as the basis functions. The unknown normalized impedance, $\zeta$, is educed by an iterative process that determines the resistance, $\theta$, and reactance, $\chi$, that reproduce the acoustic wall pressures at the microphone locations. This is achieved by minimizing the objective function

$$
F(\theta, \chi)=\sum_{n=1}^{n w a l l}\left\|\left\{R_{\text {dif }}\right\}\right\|_{L 2},\left\{R_{\text {dif }}\right\}=\left\{\begin{array}{c}
p_{\text {mic }}\left(x_{1}\right)-p_{\text {pred }}\left(x_{1}\right) \\
p_{\text {mic }}\left(x_{2}\right)-p_{\text {pred }}\left(x_{2}\right) \\
\vdots \\
p_{\text {mic }}\left(x_{\text {nwall }}\right)-p_{\text {pred }}\left(x_{\text {nwall }}\right)
\end{array}\right\}
$$

where $n$ wall is the number of microphone locations (i.e., 53 for this investigation, as mentioned in Section III) and $\left\|\left\{R_{d i f}\right\}\right\|_{L 2}$ denotes the $L_{2}$-norm of the complex residual error vector between the microphone data $\left(p_{m i c}\right)$ and predicted value $\left(p_{\text {pred }}\right)$.

\section{Results and Discussion}

\section{A. Uniform Flow}

Based on measured GFIT data, the eduction methodologies described in Section [II] were used to educe the impedance and/or axial wavenumber of the test liners for each test condition. Employing the repeated test points, $95 \%$ confidence intervals were also generated where appropriate. However, before identifying some the effects of axial wave number uncertainty, results for single instances provide excellent examples of the aforementioned source location (upstream or downstream) effects. Single instance educed impedances using the PBE approach with upstream and downstream source $(120 \mathrm{~dB})$ measurements for cases with no mean flow $\left(M_{o}=0.0\right)$ are first presented in Figures 3 and 4 . Specifically, the educed normalized resistance and reactance values for the CT57 liner are provided in Figure 3 . As expected, the results for the upstream (U) and downstream (D) compare very well in the absence of mean flow effects. In addition, the resistance results illustrate an interesting behavior of the CT57 liner, as a large increase in resistance is observed around the antiresonance $(f \approx 2100 \mathrm{~Hz}$ ). It should be noted that similar results were obtained for the higher source level in this and other instances. Therefore, all subsequent results are presented for the same $120 \mathrm{~dB}$ source level.

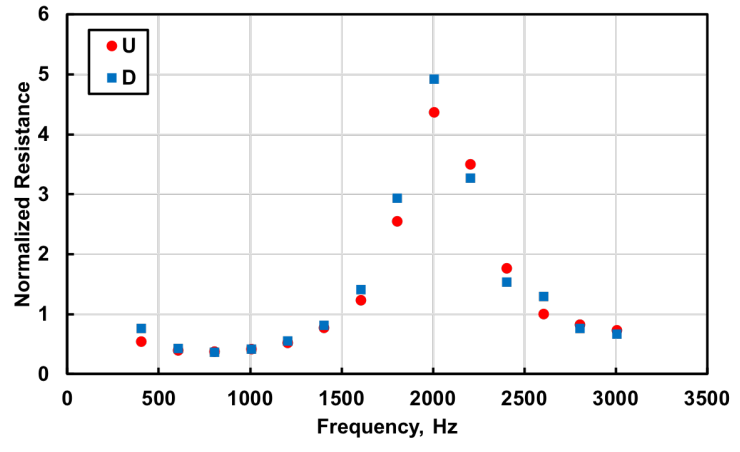

(a) Normalized Resistance

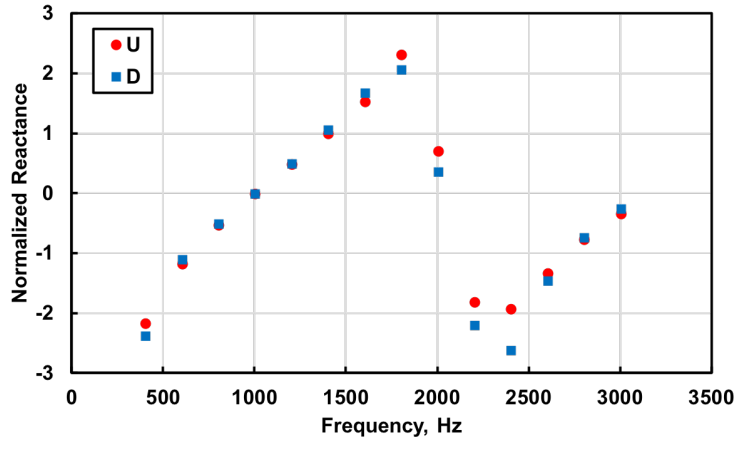

(b) Normalized Reactance

Figure 3: Educed impedance for the CT57 liner at Mach 0.0. Results for upstream and downstream source locations are represented with red circles and blue squares, respectively. 
Figure 4 shows the source location effects to be similarly negligible for the GE03 liner in the absence of mean flow. However, when a uniform flow (centerline Mach number of $0.3, M_{\text {ave }}=0.256$ ) is included by setting $M_{o}=M_{\text {ave }}$ in Eq. 4, the situation changes. As seen in Figure 5, the educed impedances for the CT57 liner with upstream and downstream sources are quite different. The discussion in Section [ alluded to this type of unexpected behavior, which has increased interest in impedance boundary conditions and the effects of flow profile and axial wavenumber uncertainties on educed impedances using such indirect methods.

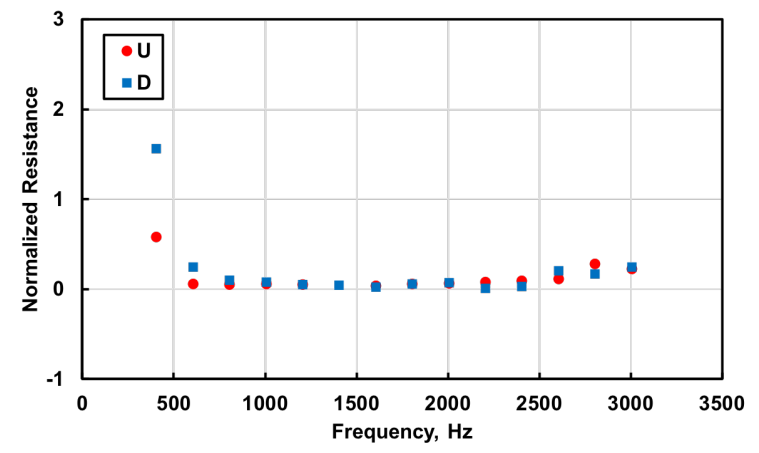

(a) Normalized Resistance

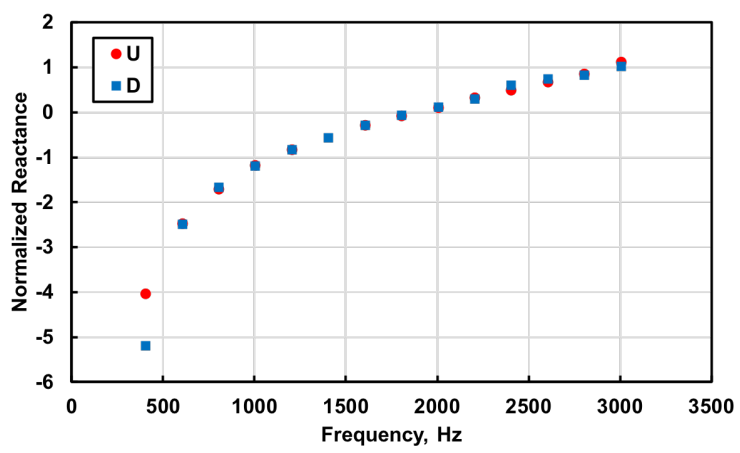

(b) Normalized Reactance

Figure 4: Educed impedance for the GE03 liner at Mach 0.0. Results for upstream and downstream source locations are represented with red circles and blue squares, respectively.

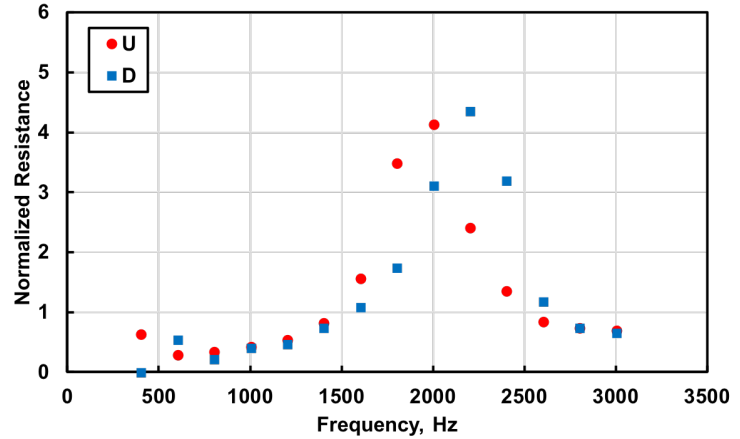

(a) Normalized Resistance

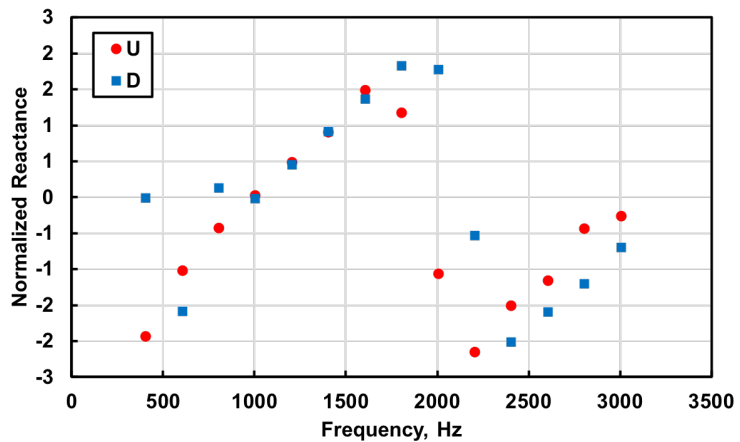

(b) Normalized Reactance

Figure 5: Educed impedance for the CT57 liner at a centerline Mach number of 0.3. Results for upstream and downstream source locations are represented with red circles and blue squares, respectively.

As the measurements in the GFIT are obviously obtained in the presence of a three-dimensional sheared mean flow, the value of the input uniform Mach number appears to be a reasonable starting point for further investigation. In general, when uniform flow is considered, an average Mach number based on the measured profiles mentioned in Section [I] is used. However, the actual value used may be subject to interpretation. For example, Eversman and Gallman ${ }^{17}$ proposed including the uniform flow Mach number as an optimization parameter in their impedance eduction process. In addition, the actual GFIT lower wall microphone locations are not located on the duct centerline. Thus, it may be more appropriate to use a uniform mean flow Mach number value that is less than the overall average. In light of this, the value of the input uniform Mach number was progressively reduced to identify general trends in the educed impedance values. If proven to be an effective modification, this would ideally be a more explicit initial step in the eduction process. However, a more ad hoc approach, in which the input Mach number was reduced until the CT57 reactance curves showed consistent antiresonance values (i.e., negative going zero crossing at $f \approx 2100 \mathrm{~Hz}$ ) seemed appropriate for this preliminary investigation. The results are presented in Figure 6 as the uniform Mach number was decreased from $M_{o}=0.256\left(\mathrm{M}^{*} 1.00\right)$ to a value of $M_{o}=0.236\left(\mathrm{M}^{*} 0.92\right)$, with an intermediate value included to illustrate the trend. As seen in the figure, the educed impedance values for upstream (U, $\left.M^{*} 0.92\right)$ and downstream $\left(\mathrm{D}, \mathrm{M}^{*} 0.92\right)$ sources are quite consistent. The peak in the resistances near antiresonance are well aligned, as are the 
overall reactance curves.

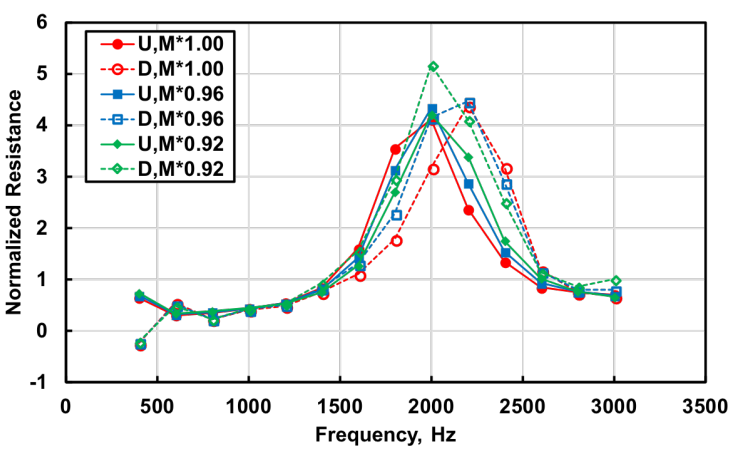

(a) Normalized Resistance

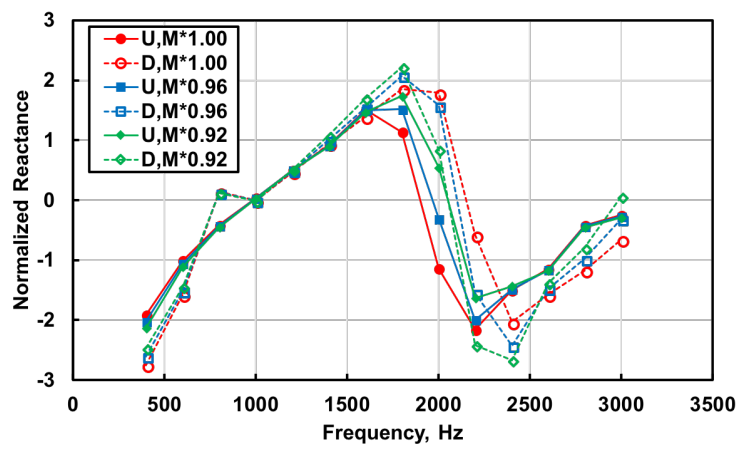

(b) Normalized Reactance

Figure 6: Educed impedance for the CT57 liner at a centerline Mach number of 0.3. Results for upstream and downstream source locations are represented with closed and open symbols, respectively.

To further investigate this behavior, the same Mach number value, $M=0.236\left(\mathrm{M}^{*} 0.92\right)$, was used in the eduction process for the GE03 liner and the results are shown in Figure 7. While the impedance curves are more benign than the CT57 case, it is clear that the educed impedances are much more consistent with the reduced Mach number, particularly at higher frequencies. In fact, with the original Mach number value the reactance curve for the downstream source (D, $\mathrm{M}^{*}$ 1.00) contains a nonphysical flattening (even downward trend) above $1600 \mathrm{~Hz}$. This feature is not present for the upstream source $\left(\mathrm{U}, \mathrm{M}^{*} 1.00\right)$ and the two curves clearly diverge. However, with the reduced Mach number ( $\left.\mathrm{M}^{*} 0.92\right)$, the curves coalesce to a physically realistic result.

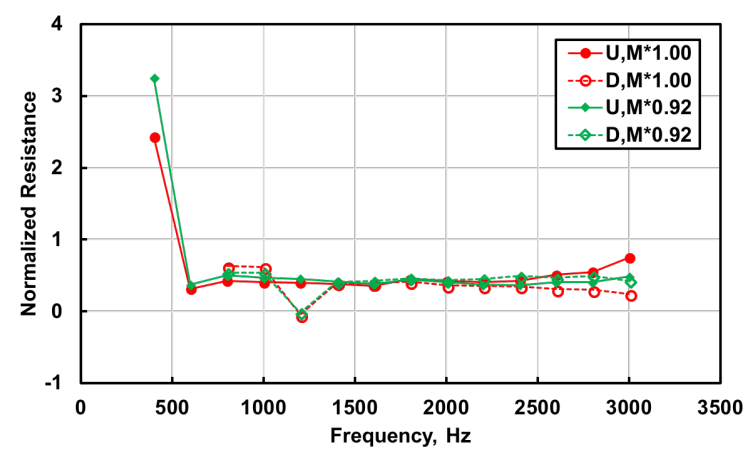

(a) Normalized Resistance

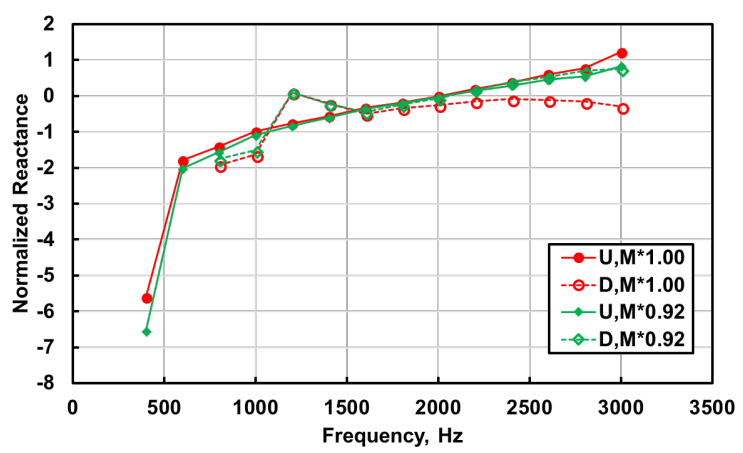

(b) Normalized Reactance

Figure 7: Educed impedance for the GE03 liner at a centerline Mach number of 0.3. Results for upstream and downstream source locations are represented with closed and open symbols, respectively. Note that impedance values at 400 and $600 \mathrm{~Hz}$ for the downstream source are not included due to anomalous behavior.

While the reduction in Mach number provides more consistent results for upstream and downstream sources, there are specific frequencies at which anomalies remain. The differences in reactance values at $800 \mathrm{~Hz}$ for the CT57 liner in Figure 6 and at $1200 \mathrm{~Hz}$ for the GE03 liner in Figure 7 are examples. In light of this, the 95\% confidence intervals on the axial wavenumbers discussed above were used to explore this further. For example, the real and imaginary parts of the axial wavenumber (with 95\% confidence intervals denoted by error bars) educed for the CT57 liner at a centerline Mach number of 0.3 are presented in Figure 8 . It can be seen that wavenumber values are generally quite consistent. However, there are large uncertainties in the values at $800 \mathrm{~Hz}$ for a downstream source. Referring back to Figure 6, this appears to contribute to anomalous reactance values for a downstream source regardless of input Mach number.

To further illustrate this behavior, the real and imaginary parts of the axial wavenumber (with $95 \%$ confidence intervals denoted by error bars) educed for the GE03 liner at a centerline Mach number of 0.3 are presented in Figure 9 Again, the wavenumber values are fairly consistent with large uncertainties at 1200 and $1400 \mathrm{~Hz}$ for the downstream 
source. There is also some increased uncertainty at $1600 \mathrm{~Hz}$ for the upstream source. However, these appear to be a lesser concern, as the associated confidence intervals are much smaller than those for the downstream anomalies. This is further supported by the results in Figure 7, which show anomalies in the resistance and reactance curves at 1200 and $1400 \mathrm{~Hz}$. Thus, it appears that uncertainties in the wavenumber may be associated with erroneous impedance values.

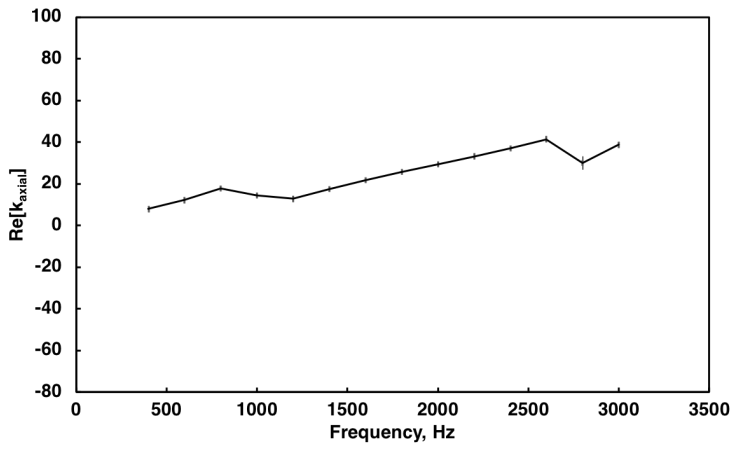

(a) Upstream Source: $\operatorname{Re}\left[k_{m n}\right]$

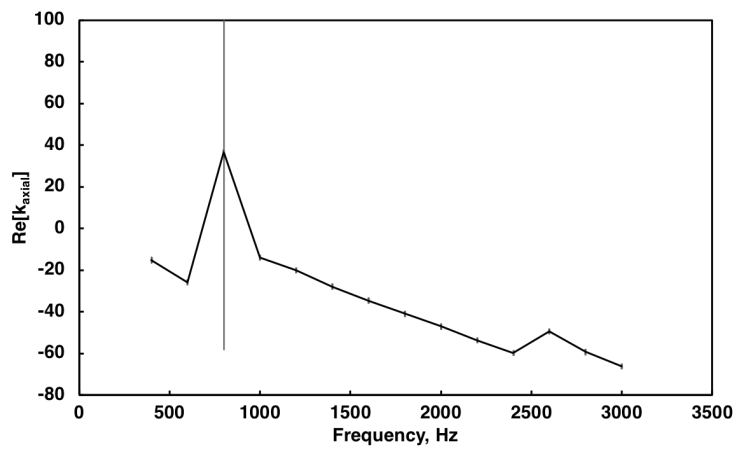

(c) Downstream Source: $\operatorname{Re}\left[k_{m n}\right]$

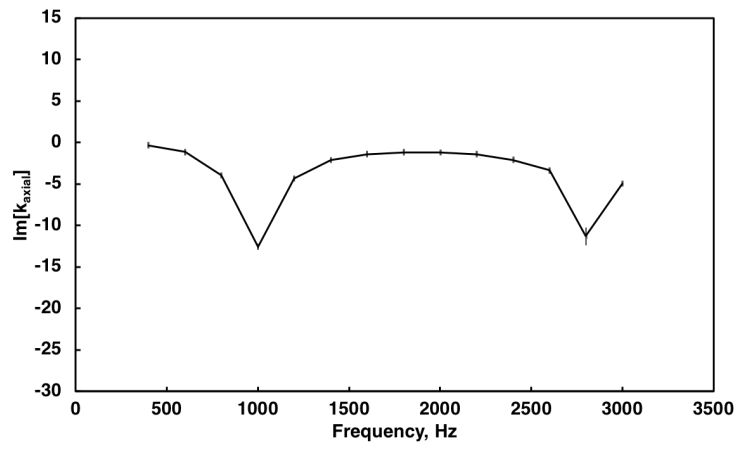

(b) Upstream Source: $\operatorname{Im}\left[k_{m n}\right]$

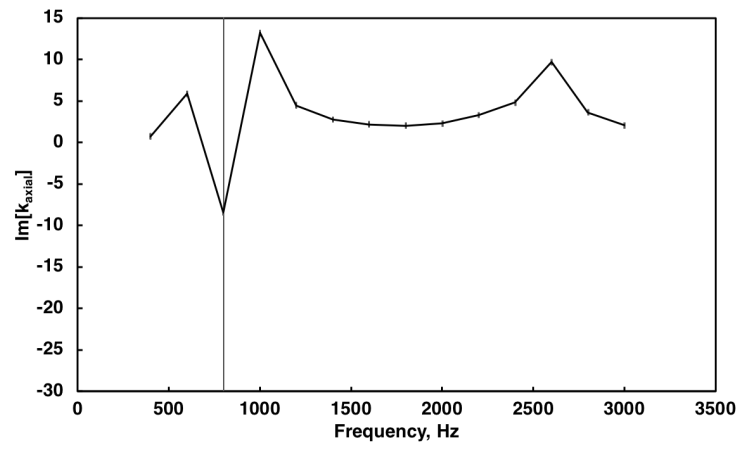

(d) Downstream Source: $\operatorname{Im}\left[k_{m n}\right]$

Figure 8: Real and imaginary parts of the complex axial wavenumber $\left(k_{m n}\right)$ educed for the CT57 liner at a centerline Mach number of 0.3. Error bars represent the $95 \%$ confidence intervals based on 11 datasets.

To close the discussion of impedance eduction with uniform flow, results for the two test liners using the CHE approach are also presented. Recall that this method entails numerical solution of the convected Helmholtz equation and does not rely on the axial wavenumber as input. Instead, the uniform mean flow Mach number and lower wall pressure measurements are provided and candidate impedance values are iterated upon until the objective function (based on the difference between measured and predicted wall pressures) is reduced to a specified tolerance. Therefore, the uniform flow Mach number and measured lower wall pressures (also used to educe axial wavenumbers in the PBE approach) were used as input. The results for the CT57 liner are provided in Figure 10 using the same nomenclature as that for the PBE approach. Similar to the PBE results in Figure 6, the modified input Mach number (M*0.92) leads to much greater consistency between the upstream and downstream source results. Again, the peaks in the resistances near antiresonance are well aligned, as are the overall reactance curves. Some increased discrepancies are visible around $800 \mathrm{~Hz}$ that may coincide with the wavenumber uncertainty in this frequency range. There are also considerable anomalies above $2600 \mathrm{~Hz}$. However, this is consistent with previous results for this approach, as this implementation assumes plane waves in the hardwall sections and higher order modes are cuton above $2600 \mathrm{~Hz}$ in the GFIT at this Mach number.

Similar conclusions may be drawn from the GE03 results shown in Figure 11. Again, the educed impedances are much more consistent with the reduced Mach number. In addition, with the original Mach number value the reactance curve for the downstream source (D, $\left.M^{*} 1.00\right)$ contains an even more pronounced nonphysical flattening above 1600 Hz. This feature is not present for the upstream source (U, $\left.\mathrm{M}^{*} 1.00\right)$ and the two curves clearly diverge even more than the PBE case. However, with the reduced Mach number $\left(\mathrm{M}^{*} 0.92\right)$, the curves again coalesce to a physically realistic result. It is also interesting to note slight anomalies near $1200-1400 \mathrm{~Hz}$ that may be linked to wavenumber 


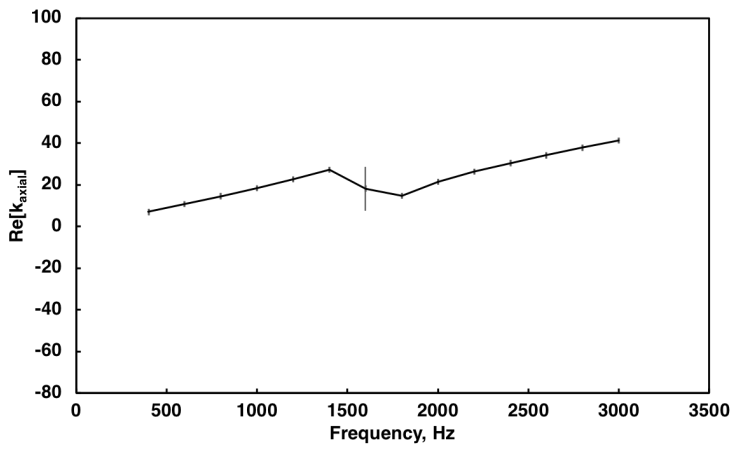

(a) Upstream Source: $\operatorname{Re}\left[k_{m n}\right]$

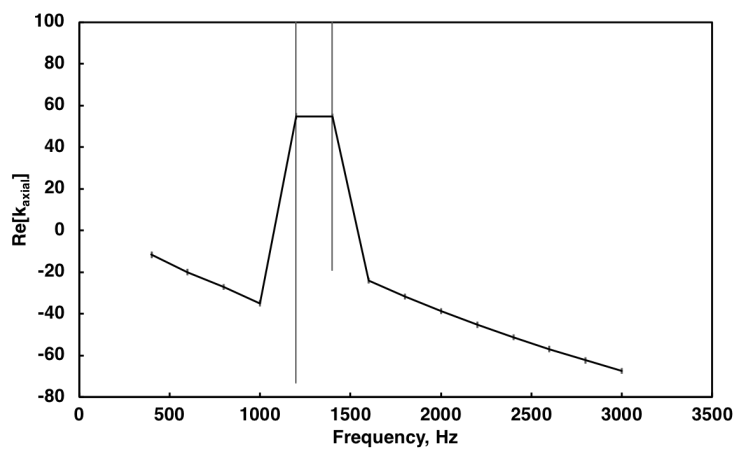

(c) Downstream Source: $\operatorname{Re}\left[k_{m n}\right]$

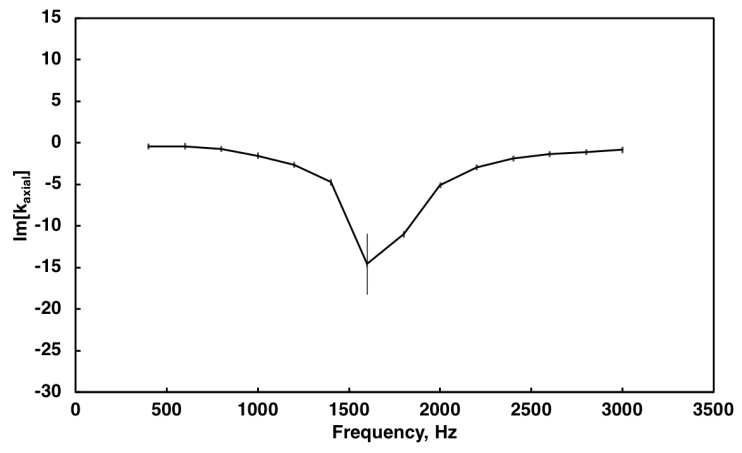

(b) Upstream Source: $\operatorname{Im}\left[k_{m n}\right]$

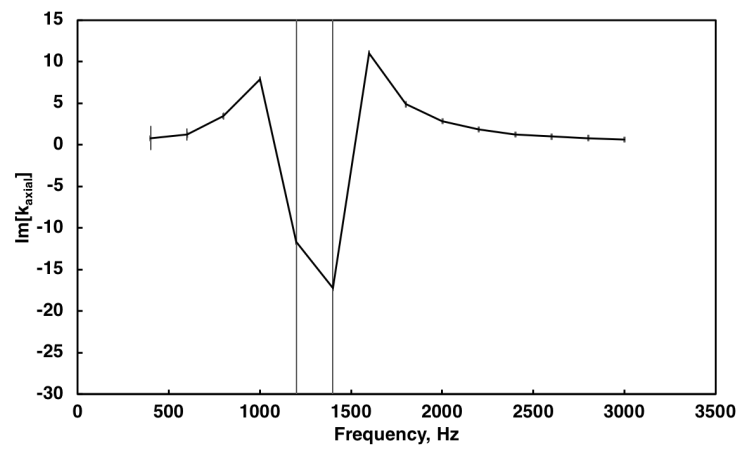

(d) Downstream Source: $\operatorname{Im}\left[k_{m n}\right]$

Figure 9: Real and imaginary parts of the complex axial wavenumber $\left(k_{m n}\right)$ educed for the GE03 liner at a centerline Mach number of 0.3. Error bars represent the $95 \%$ confidence intervals based on 11 datasets.

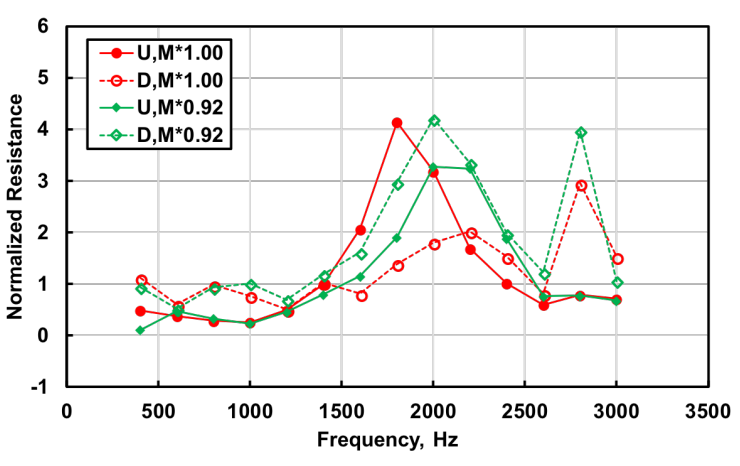

(a) Normalized Resistance

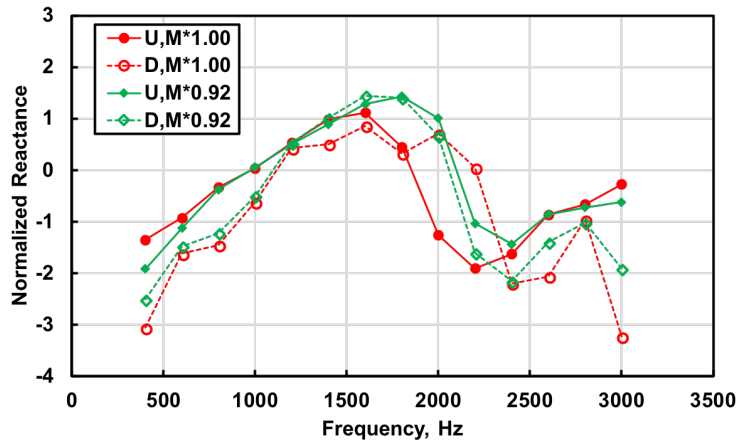

(b) Normalized Reactance

Figure 10: Educed impedance for the CT57 liner at a centerline Mach number of 0.3. Results for upstream and downstream source locations are represented with closed and open symbols, respectively. 
uncertainties discussed previously.

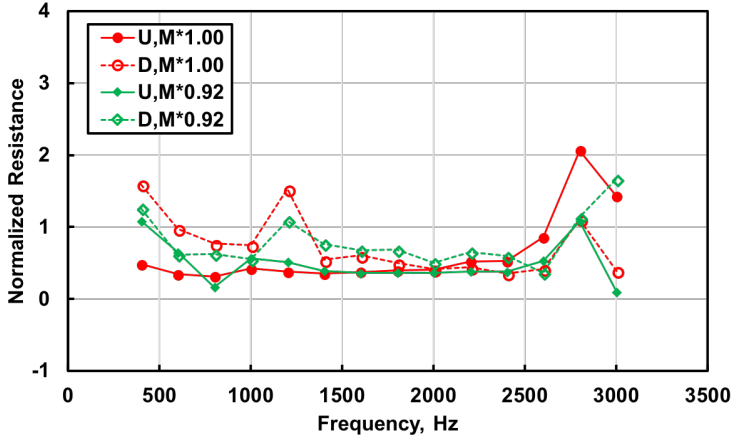

(a) Normalized Resistance

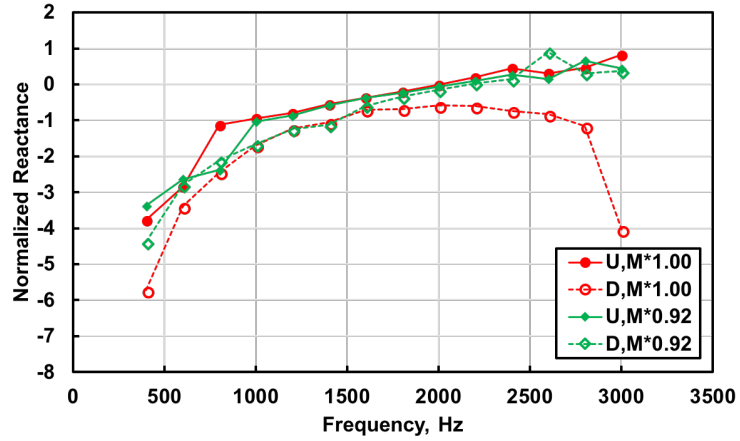

(b) Normalized Reactance

Figure 11: Educed impedance for the GE03 liner at a centerline Mach number of 0.3. Results for upstream and downstream source locations are represented with closed and open symbols, respectively.

\section{B. Shear Flow}

Although space constraints preclude much more than a cursory investigation of the above observations related to the PBE approach using shear flow, a brief consideration of flow profile scaling may be of interest. As mentioned in Section III. 2D flow profiles were acquired upstream and downstream of the test section from which centerline (1D) profiles and average Mach number values could be extracted. As an example, flow profile measurements for a centerline Mach number of 0.3 are shown in Figure 12. In order to use this information in the shear flow PBE approach (see Eqs. 1. 33, a fit of the extracted centerline $(z=1.0$ ") mean flow profile (for the appropriate liner) of the form

$$
M_{o}(\tilde{y})=\lambda_{1}(1-\tilde{y}) \ln \left[1+\lambda_{2} \tilde{y}-\lambda_{3} \tilde{y}^{2}\right]+\lambda_{4} \tilde{y} \ln \left[1+\lambda_{5}(1-\tilde{y})-\lambda_{6}(1-\tilde{y})^{2}\right]
$$

is first computed. Here, $\tilde{y}=y / H$ and $\lambda_{n}$ are constant coefficients determined by minimizing the $L_{2}$-norm of the error between the 'fit' and measured values over the range of measurement locations. The functional form of Eq. 6 has been used in previous studies and provided results that match the measured data quite well. However, alternative forms may be equally (or more) valid and would obviously lead to different values of $M_{o}^{\prime}$. Exploration of the effects of alternate forms of the mean flow profile fit is certainly of interest, and this is intended to be the focus of future studies. In this work, a simple sensitivity study is employed to gain insight into some of the effects of flow profile shape.

The Mach number profile obtained from Eq. 6 was used to perform preliminary eduction with the PBE shear flow approach and, similar to the uniform flow cases, upstream and downstream results were inconsistent. Therefore, while not expected to be as applicable as in the uniform flow cases, a similar ad hoc approach was used in which the mean flow profile was scaled until the CT57 reactance curves showed consistent antiresonance values (i.e., negative going zero crossing at $f \approx 2100 \mathrm{~Hz}$ ). The applicability of this approach may be suspect in this application for a number of reasons, including a mismatch in the centerline Mach number and modification of $M_{o}^{\prime}$ by only a constant factor. However, it may generate some basic insights into general flow profile modifications that lead to consistent results for upstream and downstream source locations. Bearing this in mind, the results of this cursory study are provided in Figure 13 from which one may glean a few observations of interest. First and foremost, the upstream and downstream results may be made more consistent by a simple scaling of the profile. However, a large scaling factor was required, which is contrary to the behavior for the uniform flow case. This is likely due, in large part, to the inclusion of $M_{o}^{\prime}$ as an additional parameter and will be investigated in future work. Additionally, based on the behavior of the reactance near $800 \mathrm{~Hz}$, the uncertainty in wavenumber appears to have a similar effect as in the uniform flow case. Overall, this brief analysis provides some indication that, while certainly more complex than in the uniform flow cases, modification of the mean flow profile offers some hope to improving consistency in educed impedance values for upstream and downstream source locations. 


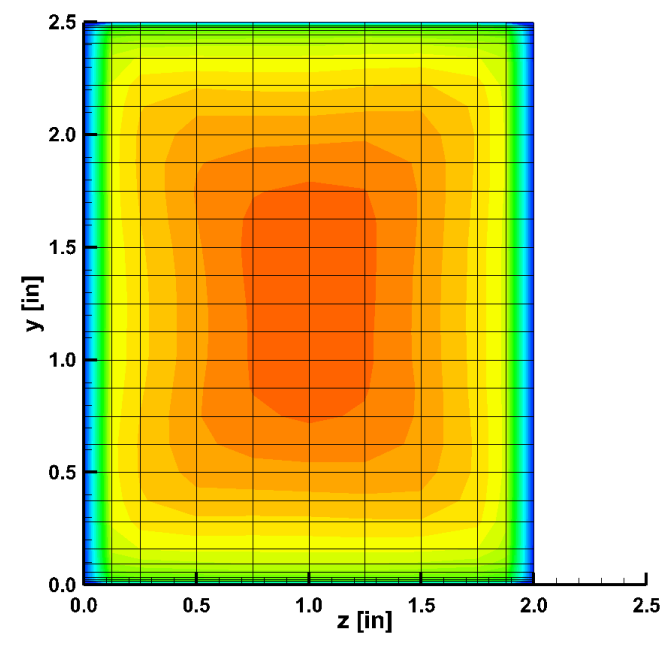

(a) Upstream axial location

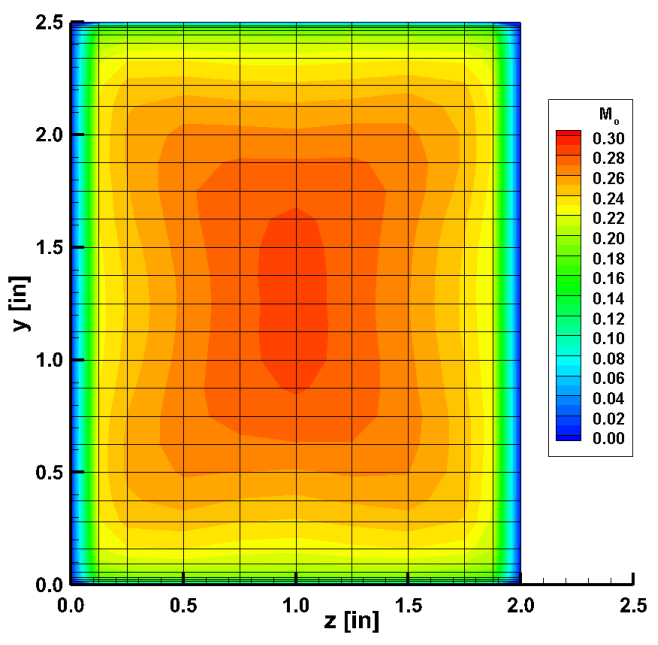

(b) Downstream axial location

Figure 12: Measured flow profiles at axial locations upstream and downstream of the GE03 liner with a centerline Mach number of 0.3. Grid lines represent the 9 horizontal and 31 vertical measurement locations.

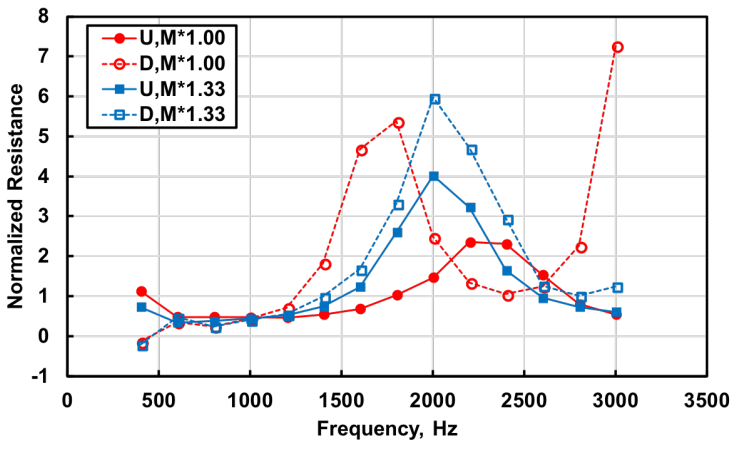

(a) Normalized Resistance

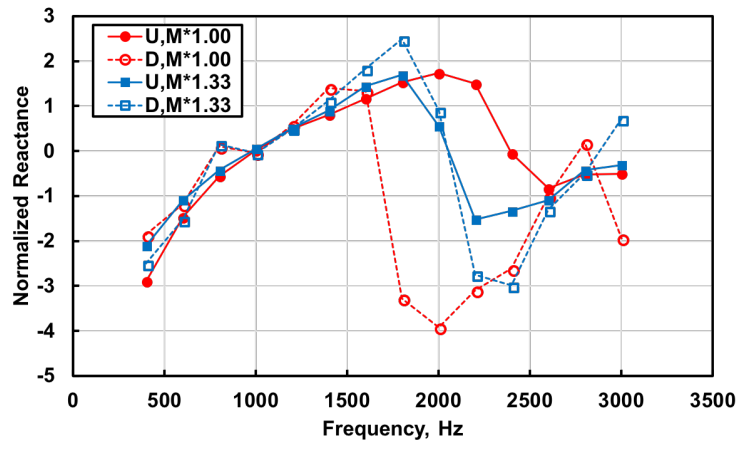

(b) Normalized Reactance

Figure 13: Educed impedance for the CT57 liner with a shear flow profile and centerline Mach number of 0.3. Results for upstream and downstream source locations are represented with closed and open symbols, respectively. 


\section{Concluding Remarks}

This paper describes the testing and subsequent eduction processes for two acoustic liners in the GFIT using upstream and downstream acoustic sources. The uniform flow results for the PBE and CHE approaches demonstrate the importance of setting a correct Mach number value in obtaining consistent educed impedances for upstream and downstream sources. In fact, the consistency of results over the two source locations was greatly improved by slightly decreasing the uniform flow Mach number from the initial average value. In addition, the effect of wavenumber uncertainty was also illustrated as large uncertainties for the downstream source correlated well with differences in the educed impedances, even with modified uniform flow Mach number. Finally, while less straightforward than in the uniform flow case, it appears that modification of the mean flow profile may also improve consistency of results for upstream and downstream results when shear flow is included in the PBE method.

Future work will include the establishment of a rigorous method for selecting the uniform flow Mach number for use in the aforementioned indirect impedance eduction methods. Exploration of the effects of alternate forms of the mean flow profile, and $M_{o}^{\prime}$ in particular, for shear flow cases will also be considered in subsequent studies.

\section{Acknowledgments}

The authors would like to thank Martha Brown, Brian Howerton, and Max Reid for obtaining the GFIT data used in this study. This work is also part of the NASA/ONERA collaborative agreement on aircraft noise reduction. The NASA portion of this research was funded by the Advanced Air Transport Technology (AATT) Project of the NASA Advanced Air Vehicles Program (AAVP).

\section{References}

${ }^{1}$ Jones, M. G. and Watson, W. R., "On the Use of Experimental Methods to Improve Confidence in Educed Impedance," AIAA Paper 20112865, 2011.

${ }^{2}$ Watson, W. R., Tracy, M. B., Jones, M. G., and Parrott, T. L., "Impedance Eduction in the Presence of Shear Flow," AIAA Paper 2001-2263, 2001.

${ }^{3}$ Jones, M. G., Watson, W. R., and Nark, D. M., "Effects of Flow Profile on Educed Acoustic Liner Impedance," AIAA Paper 2010-3763, 2010.

${ }^{4}$ Pridmore-Brown, D. C., "Sound Propagation in a Fluid Flowing Through an Attenuating Duct," Journal of Fluid Mechanics, Vol. 4, 1958, pp. 393-406.

${ }^{5}$ Ingard, U., "Influence of fluid motion past a plane boundary on sound reflection, absorption, and transmission," Journal of the Acoustical Society of America, Vol. 31, 1959, pp. 1035-1036.

${ }^{6}$ Myers, M. K., "On the Acoustic Boundary Condition in the Presence of Flow," Journal of Sound and Vibration, Vol. 71, No. 3, 1980, pp. 429-434.

${ }^{7}$ Renou, Y. and Auregan, Y., "Failure of the Ingard-Myers boundary condition for a lined duct: An experimental investigation," Journal of the Acoustical Society of America, Vol. 130, No. 1, July 2011, pp. 52-60.

${ }^{8}$ Watson, W. R. and Jones, M. G., "A Comparative Study of Four Impedance Eduction Methodologies Using Several Test Liners," AIAA Paper 2013-2274, 2013.

${ }^{9}$ Weng, C., Schulz, A., Ronneberger, D., Enghardt, L., and Bake, F., "Impedance eduction in the presence of turbulent shear flow using the linearized Navier-Stokes equations," AIAA Paper 2017-3182, 2017.

${ }^{10}$ Zhou, L. and Bodén, H., "A systematic uncertainty analysis for liner impedance eduction technology," Journal of Sound and Vibration, Vol. 356, 2015, pp. 86-99.

${ }^{11}$ Spillere, A. M. N., Cordioli, J. A., and Bodén, H., "On the effect of boundary conditions on impedance eduction results," AIAA Paper 2017-3185, June 2017.

${ }^{12}$ Bodén, H., Cordioli, J. A., Spillere, A. M. N., and Serrano, P. G., "Comparison of the effect of flow direction on liner impedance using different measurement methods," AIAA Paper 2017-3184, June 2017.

${ }^{13}$ Watson, W. R. and Jones, M. G., "Comparison of a Convected Helmholtz and Euler Model for Impedance Eduction in Flow," AIAA Paper 2006-2643, 2006.

${ }^{14}$ Watson, W. R. and Jones, M. G., "Impedance Eduction in Ducts with Higher-Order Modes and Flow," AIAA Paper 2009-3236, 2009.

${ }^{15}$ Jones, M. G. and Watson, W. R., "Explanation of Anomalous Behavior Observed in Impedance Eduction Techniques Using Measured Data," AIAA Paper 2010-3766, 2010.

${ }^{16}$ Watson, W. R., Carpenter, M. H., and Jones, M. G., "Performance of Kumaresan and Tufts Algorithm in Liner Impedance Eduction with Flow," AIAA Journal, Vol. 53, No. 4, April 2015, pp. 1091-1102.

${ }^{17}$ Eversman, W. and Gallman, J. M., "Impedance Eduction with an Extended Search Procedure," AIAA Journal, Vol. 49, No. 9, Sept. 2011, pp. 1960-1970. 